\title{
Guía práctica clínica sobre los aspectos deontológicos de la medicina legal
}

\section{Clinical practice gidelines in deontological aspects of legal medicine}

Recibido: $14 / 07 / 2021$

Aceptado: 17/08/2021

\author{
Berta Karina Zuñiga de la Rosa \\ Carrera Médico y Cirujano \\ Universidad de San Carlos de Guatemala, \\ bertakarinaz.dr@gmail.com
}

\section{Referencia del artículo}

Zuñiga de la Rosa, B. K. (2021). Guía práctica clínica sobre los aspectos deontológicos de la medicina legal . Revista Diversidad Científica, 1(1). 141-149.

DOI: https://doi.org/10.36314/diversidad.v1i1.15

\section{Resumen}

OBJETIVO: el objetivo de la investigación fue elaborar una Guía Práctica Clínica sobre los Aspectos Deontológicos de la Medicina Legal con la mejor evidencia científica y legal posible destinada a estudiantes de la Carrera de Médico y Cirujano del Centro Universitario de Oriente de la Universidad de San Carlos de Guatemala. MÉTODO: se utilizó la metodología de investigación descriptivo documental, el desarrollo de temas se basó en literatura científica, legal y normas jurídicas vigentes guatemaltecas de interés médico. La revisión de la guía estuvo a cargo de cuatro profesionales expertos, tres abogados y notarios y un médico y cirujano con maestría en medicina forense. RESULTADOS: se elaboró una guía que desarrolla trece capítulos con temas deontológicos que se pueden utilizar para evitar conflictos medicolegales. CONCLUSIÓN: en el pensum de estudios actual de la carrera de Médico y Cirujano del Centro Universitario de Oriente de la Universidad de San Carlos de Guatemala no se desarrollan temas de medicina legal enfocados a la deontología médica, por lo que con evidencia científica y la legislación vigente guatemalteca se elaboró la primera guía sobre temas deontológicos que servirá como fuente bibliográfica verídica, confiable y actualizada para sus estudiantes.

Palabras clave: guía práctica clínica basada en evidencia, medicina legal, deontología médica 


\begin{abstract}
OBJECTIVE: the objective of the research was to elaborate a Clinical Practical Guide on the Deontological Aspects of Legal Medicine with the best possible scientific and legal evidence for the students of the medical school of Centro Universitario de Oriente, of the Universidad de San Carlos de Guatemala. METHOD: the descriptive documentary research methodology was used, the development of topics was based on scientific and legal literature and current Guatemalan legal norms of medical interest. The guideline was reviewed by four expert professionals, three lawyers and notaries, and a doctor and surgeon with a master's degree in forensic medicine. RESULTS: a guide was developed that develops thirteen chapters with ethical issues that can be used to avoid medico-legal conflicts. CONCLUSION: in the current curriculum of the medical school of Centro Universitario de Oriente, of the Universidad de San Carlos de Guatemala, legal medicine topics focused on medical deontology are not developed, so with scientific evidence and current legislation in Guatemala, the first guide on deontological issues was elaborated that will serve as a true, reliable and updated bibliographic source for its students.
\end{abstract}

Keywords: clinical practical guide based on evidence, legal medicine, medical deontology 


\section{Introducción}

Las guías de práctica clínica son recomendaciones para el médico basadas en evidencia científica que lo orientan de acuerdo con la situación clínica del paciente, sobre que conducta debe tomar para brindar la mejor atención integral (Casariego Vales y Costa Ribas, 2019, párr. 9).

La medicina legal es la disciplina que vincula los conocimientos de la medicina y del derecho, para estudiar las situaciones éticas, sociales o jurídicas en las que el médico puede estar sujeto debido al ejercicio de su profesión. La deontología médica es una de las disciplinas relacionadas directamente con la medicina legal; aplica sus conocimientos para establecer cuál debe ser el comportamiento del profesional médico según los principios éticos y legales en las diversas situaciones que se le puedan presentar.

En la carrera de Médico y Cirujano del Centro Universitario de Oriente de la Universidad de San Carlos de Guatemala no se han elaborado guías sobre deontología médica o medicina legal y el contenido programático del curso de patología incluye temas como: aspectos médico legales en la práctica médica, necropsia clínica y médico legal, elaboración de protocolo de necropsias y certificación médica de defunción, informe médico legal, tanatología forense, fenómenos transformativos, lesiones transformativos y lesiones físicas (Marroquín Estrada, 2019, pág. 7), que resultan no ser lo suficiente amplios para la formación deontológica profesional de los futuros médicos.

En Guatemala, "la ignorancia no exime a la persona de su responsabilidad" de acuerdo con lo regulado en la Ley del Organismo Judicial (Congreso de la República de Guatemala, 1989, pág. 1) y además de la legislación vigente son pocas las fuentes literarias que los estudiantes pueden consultar sobre deontología médica, por lo que la elaboración de una guía de práctica clínica que incluya temas como la práctica médica, la relación del médico con el paciente, relación del médico con otros colegas, instituciones y profesiones afines, la eutanasia, los honorarios médicos, la responsabilidad médica, el secreto médico, la documentación médica, el consentimiento informado, la investigación biomédica, donación de órganos y la tarea pericial, es el aporte basado en evidencia científica actual sobre la regulación de los deberes y obligaciones básicos e imprescindibles que todo estudiante de la Carrera de Médico y Cirujano del Centro Universitario de Oriente de la Universidad de San Carlos de Guatemala debe conocer para ejercer en su práctica hospitalaria y carrera profesional. 


\section{Contenido}

La Guía Práctica Clínica sobre los Aspectos Deontológicos de la Medicina Legal nace de la importancia que tiene la deontología para la medicina, tema que, al no ser tratado con profundidad durante la formación académica del futuro médico y no existir en Guatemala una guía que aborde este tema de una manera ordenada y enfocada a estudiantes, estos carecen de conocimientos fundamentales sobre la misma.

En su gran mayoría los estudiantes e incluso algunos profesionales no podrían discernir ¿Qué? legalmente es aceptable en su práctica médica, colocándolos en riesgo de cometer alguna conducta contraria a las normas jurídicas, de manera que esta guía realiza una recopilación de distintas fuentes bibliográficas actuales y contiene distintos puntos clave que se pueden utilizar para evitar conflictos medicolegales.

La guía inicia con el capítulo denominado generalidades de la medicina legal, que incluye definiciones de algunos autores nacionales y extranjeros. Entre los autores latinos es común hablar de medicina legal, mientras que los anglosajones prefieren denominarla medicina forense. Otros autores la han denominado medicina legal y forense, cuestiones medicolegales, jurisprudencia médica, medicina legal judicial o medicina legal y toxicología (Patitó, 2000, pág. 33).

Para García-Garduza (2014), "la medicina legal comprende muchas disciplinas que permiten conocer, analizar e interpretar correctamente las normas jurídicas en las que se establecen conceptos o razonamientos que involucran el ejercicio de la medicina" entre ellas la deontología médica, que es la disciplina que delimita los deberes y obligaciones de los profesionales médicos (párr. 35).

La amplitud y diversidad de temas medicolegales permite a los autores organizarlos de forma variada. Jose Ángel Patitó los clasifica de la siguiente manera: deontología médica, patología forense, criminalística medicolegal, sexología medicolegal, tocoginecología medicolegal, psiquiatría medicolegal, medicina legal social (Patitó, 2000, pág. 34, 35).

El segundo capítulo desarrolla el tema de la deontología en el área médica, y define que "la deontología médica también es conocida como derecho médico o jurisprudencia médica e incluye los deberes y obligaciones de los médicos vinculados con las leyes (ejercicio de la medicina, responsabilidad médica, secreto médico, 
documentación médica, honorarios médicos, labor médico legal o tarea pericial) y con la ética (bioética, investigación y consentimiento informado en medicina, eutanasia y los comités de ética)" (Patitó, 2000, pág. 34).

Contiene también información acerca de los principios éticos en la medicina (principio de no maleficiencia, principio de beneficiencia, principio de equidad, principio de autonomía, principio de confidencialidad, principio de respeto, principio de honestidad, principio de solidaridad y el principio de lealtad).

En el tercer capítulo denominado, el médico y las normas jurídicas, se desarrollan temas como la jerarquía normativa que determina que "las normas de un sistema jurídico pueden ser del mismo o distinto nivel; si son del mismo nivel se produce una relación de coordinación y si las normas son de distinto nivel se producen relaciones de supra o de subordinación" (Torres Moss, 1998, pág. 72).

En este capítulo también se incluye las normas jurídicas guatemaltecas que son de importancia médica, tales como, la Constitución Política de la República de Guatemala, Código Civil, Código Procesal Civil y Mercantil, Código Penal, Código Procesal Penal, Código de Salud, Ley del Organismo Judicial, Ley de Colegiación Obligatoria, Código Deontológico, Estatutos del Colegio de Médicos y Cirujanos de Guatemala y la Normativa para Estudiantes de Grado de la Carrera de Medicina en los Hospitales de la Red de Servicios de salud.

El cuarto capítulo aborda la legalidad en la actividad médica, debido a que el estudiante universitario que se gradúa de médico y cirujano debe cumplir con varios requisitos para realizar la actividad médica de forma legal. Uno de éstos es la colegiación profesional ante el Colegio de Médicos y Cirujanos de Guatemala, con la finalidad de promover el buen actuar médico, controlar su ejercicio y evitar que personas no preparadas, no autorizadas ofrezcan servicios médicos y comprometan la salud de la población.

La práctica de la medicina no debe alejarse de la ética ni ser contraria a la ley; si fuera el caso, el derecho penal se encarga de regular las actividades médicas y sancionarlas al cometerse, según la gravedad del hecho, delitos o faltas expresamente calificados en la ley. Por ello, el quinto capítulo denominado, el derecho penal en la actividad médica, presenta las conductas típicas, antijurídicas, culpables y/o punibles que pueden estar susceptibles de cometer los médicos como asesinato, homicidio, aborto, lesiones, maltrato, omisión de auxilio, delitos contra el honor, etc. 
El sexto capítulo se refiere a la responsabilidad profesional del médico, y profundiza en temas como la culpa, que según el artículo 1424 del Código Civil (1963, pág. 169) "consiste en una acción u omisión, perjudicial a otro, en que se incurre por ignorancia, impericia o negligencia, pero sin propósito de dañar". También abarca las causas que eximen la responsabilidad penal como la legítima defensa y las circunstancias que modifican la responsabilidad penal. Así mismo, contiene algunas recomendaciones para prevenir conflictos medicolegales.

"El secreto profesional es la obligación de guardar silencio sobre las cuestiones que deben ser mantenidas reservadas, es decir, que deben ser despojadas de la posibilidad de que terceras personas accedan a las mismas y de las que se ha tomado conocimiento debido a un trabajo, empleo, arte o profesión" (Patitó, 2000, pág. 99), debido a esto, se dedica el séptimo capitulo a tratar este tema.

El octavo capítulo desarrolla el tema del consentimiento informado, porque según el Código de Salud (1997, pág. 3) "todos los habitantes tienen, en relación con su salud, derecho al respeto a su persona, dignidad humana e intimidad, secreto profesional y a ser informados en términos comprensibles sobre los riesgos relacionados con la pérdida de la salud y la enfermedad y los servicios a los cuales tienen derecho". También se desarrollan sus requisitos esenciales: la capacidad, la titularidad, y la libertad, así como sus excepciones: situaciones de urgencia, pacientes incapaces, privilegio terapéutico y pruebas de $\mathrm{VIH}$.

Posteriormente, el noveno capítulo denominado las relaciones interpersonales del médico, aborda sobre los tipos de relaciones que el médico mantiene con personas individuales, tales como, pacientes, participantes, estudiantes, colegas, enfermeras, personal de intendencia y personal administrativo, o la industria farmacéutica producto del ejercicio de su profesión, con los que en todo momento es su obligación conservar una excelente conducta ética.

En el décimo capítulo se desarrolla el tema de donación de órganos y tejidos, debido a que el médico en algún momento de su práctica profesional debe orientar a sus pacientes en el tema y debería de conocer que en Guatemala sus regulaciones legales están contenidas en el Código Penal, el Código de Salud, el Código Deontológico y la Ley para la Disposición de Órganos y Tejidos Humanos.

El onceavo capítulo se trata de la investigación bioética, enfocándose en las regulaciones deontológicas relacionadas a la reproducción humana y el consen- 
timiento del paciente, así mismo, las regulaciones penales sobre inseminación o experimentación.

En algunas situaciones el médico podría actuar como perito profesional y experimentará gran responsabilidad social y jurídica para prepararse con conocimientos, habilidades y/o experiencia y ser útil en la resolución de un proceso legal, por lo que en el doceavo capítulo, denominado la tarea pericial se profundiza sobre la actividad y las cualidades del médico como perito (saber, experiencia, pericia, buena voluntad y diligencia, valor moral e independencia).

El capítulo final trata sobre los documentos médicos y su importancia legal, porque todo documento que el médico escriba, firme y/o selle está envuelto de valor legal y es necesario que el estudiante conozca, cómo debe ser su correcta elaboración. Se desarrollan y se presentan ejemplos de temas como documentos médicos (certificaciones médicas, certificaciones de salud, informes de nacimiento, certificaciones por lesiones, certificaciones por delitos sexuales, informes de defunción), el registro médico, el recetario o prescripción médica y el informe médico legal.

\section{Conclusión}

En el pensum de estudios actual de la carrera de Médico y Cirujano del Centro Universitario de Oriente de la Universidad de San Carlos de Guatemala no se desarrollan temas de medicina legal con énfasis en la deontología médica, por lo que se elaboró la primera Guía Práctica Clínica sobre los Aspectos Deontológicos de la Medicina Legal, para lo cual se utilizaron fuentes bibliográficas verídicas, confiables y actualizadas, basadas en evidencia científica y en la legislación guatemalteca vigente sobre medicina legal con realce en la deontología médica, destinada a los estudiantes de la carrera de Médico y Cirujano del Centro Universitario de Oriente de la Universidad de San Carlos de Guatemala.

\section{Declaración}

El estudio se realizó de acuerdo al Código de Ética y Buenas Prácticas COPE, con las autorizaciones del Centro Universitario de Oriente de la Universidad de San Carlos de Guatemala. 
Revista Diversidad Científica Vol. 1 No. 1 Año 2021

\section{Conflicto de intereses}

La autora declara no tener ningún conflicto de intereses.

\section{Referencias}

Casariego Vales, E. y Costa Ribas, C. (1 de febrero de 2019). ¿Que son y para qué sirven las GPC?. Fisterra. https://www.fisterra.com/guias-clinicas/ que-son-para-que-sirven-gpc/

Código Civil. Decreto Ley 106. Artículo 1424. 14 de septiembre de 1963. https://www. oas.org/dil/esp/codigo_civil_guatemala.pdf.

Código de Salud. Decreto 90-97. Artículo 3. 2 de octubre de 1997. https://asisehace. gt/media/GT_Codigo_Salud_90_97.pdf.

García-Garduza, I. (2014). Importancia de la medicina legal en la práctica médica. Revista de la Facultad de Medicina de la UNAM. 57(5), 20-31. http://www.medigraphic.com/pdfs/facmed/un-2014/un145d.pdf

Ley del Organismo Judicial. (1989). Decreto número 2-89. Artículo 3. 10 de enero de 1989. Guatemala. http://www.oas.org/juridico/pdfs/mesicic4_gtm_org.pdf

Marroquín Estrada, E. (2019). Patología: programa del curso de patología año 2019. Centro Universitario de Oriente de la Universidad de San Carlos de Guatemala.

Patitó, J. A. (2000). Medicina legal (en línea). Ediciones Centro Norte. http://evirtual. uaslp.mx/FMed/Documentos $\% 20$ compartidos/patito, $\% 20$ jose $\% 20$ angel $\% 20$ $-\% 20$ medicina\%20legal(2).pdf.

Torres Moss, J. C. (1988). Introducción al estudio del derecho. Universidad de San Carlos de Guatemala. Guatemala 


\section{Sobre la autora \\ Berta Karina Zuñiga de la Rosa}

Estudiante de la Licenciatura de Médico y Cirujano de la Universidad de San Carlos de Guatemala; estudiante de la Licenciatura en Ciencias Jurídicas y Sociales de la Universidad Mariano Gálvez de Guatemala; estudiante de la Especialización en Investigación Científica de la Escuela de Estudios de Postgrado de la Facultad de Ingeniería de la Universidad de San Carlos de Guatemala.

Copyright (c) Berta Karina Zuñiga de la Rosa

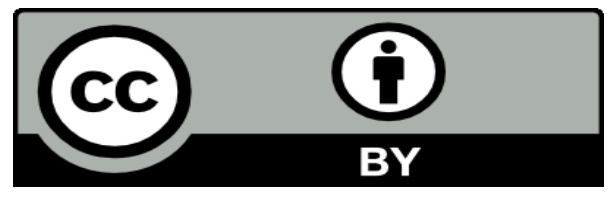

Este texto está protegido por una licencia CreativeCommons 4.0.

Usted es libre para compartir, copiar y redistribuir el material en cualquier medio o formato y adaptar el documento, remezclar, transformar y crear a partir del material para cualquier propósito, incluso comercialmente, siempre que cumpla la condición de atribución: usted debe reconocer el crédito de una obra de manera adecuada, proporcionar un enlace a la licencia, e indicar si se han realizado cambios. Puede hacerlo en cualquier forma razonable, pero no de forma tal que sugiera que tiene el apoyo del licenciante o lo recibe por el uso que hace. 